\title{
The Application of Proteomics to PK-PD Modeling and Simulation
}

Ken-ichi Sako', Hisao Haniu², Mayumi Hasegawa ${ }^{3}$, Hirohisa Doi ${ }^{3}$, Shunsuke Yano ${ }^{3}$, Yuichiro Oosawa ${ }^{3}$, Tohru Kishino ${ }^{3}$, Yoshihiko Matsuki ${ }^{4}$ Yumiko Arisue ${ }^{1}$, Takeshi Kawamura', Masayuki Kimura' ${ }^{1}$ and Yoshikazu Matsuda ${ }^{1 *}$

${ }^{1}$ Clinical Pharmaceutics Educational Center, Nihon Pharmaceutical University, 10281 Komuro, Ina-machi, Saitama 362-0806, Japan

2Institute of Carbon Science and Technology, Shinshu University, 3-1-1 Asahi, Matsumoto-shi, Nagano 390-8621, Japan

${ }^{3}$ Department of Pharmacy, Saitama Medical Center, Saitama Medical University, 1981 Kamoda, Kawagoe-shi, Saitama 350-8550, Japan

${ }^{4}$ Department of Pharmacy, Funabashi General Hospital, 4-8-28 Honcho, Funabashi-shi, Chiba 273-0005, Japan

\begin{abstract}
Pharmacokinetic-pharmacodynamic (PK-PD) modeling and simulation can be an invaluable tool for use in making crucial decisions in drug development and clinical settings, including those pertaining to compound selection, dose selection, study design, and patient population, all of which can impact the cost of development and treatment. Clinical PK-PD modeling and simulation of antimicrobial agents are possible because minimum inhibitory concentration (MIC) values can be easily measured in the hospital laboratory. However, no such easily measured clinical markers are available for many other types of drugs. In this point of view, we thought that the proteomics approach may be available for find the direct PD parameter such as drug specific biomarker. Many reports also suggest that proteomics is a promising tool in the search for drug-specific biomarker proteins that could be used in PK-PD modeling and simulation. Thus, by enabling examination of drug-induced changes in the expression of specific biomarker proteins, proteomic data could be used in PD analyses in much the same way that MICs are used in PD evaluations of antimicrobial agents.
\end{abstract}

Keywords: PK-PD modeling and simulation; Proteomics; Biomarker; Drug development; Clinical setting

\section{Introduction}

Increases in the cost of drug development have resulted in a reduction in the number of new drugs produced in recent years $[1,2]$. One potential explanation for this trend is that applied sciences related to drug development have not kept pace with advances in the basic sciences. As a result, there has been increased emphasis on the use of alternative tools that will provide answers to questions regarding drug efficacy and safety with greater speed and certainty, and at lower cost [3]. Alternative approaches to drug development include the use of adaptive trial designs [4], more extensive use of biomarkers [5], development of personalized medicines, and the use of pharmacokinetic-pharmacodynamic (PK-PD) modeling and simulations.

The use of PK-PD modeling and simulations is possible in all stages of drug development, from the preclinical discovery stage to the clinical application of drugs. PK-PD modeling and simulations can be used to direct decision making during the crucial early stages of drug development that may lead to significant cost reductions throughout the drug development process [6-8]. Although scientific evidence should direct the administration of drugs in clinical situations, these decisions are most often guided by physician experience, and this may not always serve the patient's best interest. We thus believe that wider application of PK-PD modeling and simulations would greatly assist physicians in making clinical decisions.

An understanding of the value and applicability of PK-PD modeling and simulations has typically been limited to specialists (i.e., clinical pharmacologists, clinical pharmacokineticists, biostatisticians). However, in order for PK-PD modeling and simulations to be used to its fullest potential as a tool to direct drug development and clinical drug application, the methodology must be understood and embraced by both drug development teams and medical staff. In this review, we summarize and discuss the merits of PK-PD modeling and simulation with respect to the different stages of drug development and clinical application. Typically, PK-PD modeling and simulations are employed to assess antimicrobial agents because it is relatively easy to obtain $\mathrm{PD}$ data such as minimum inhibitory concentration (MIC) values. However, PK-PD modeling and simulation of any drugs other than antimicrobial agents is difficult due to the absence of simple and/ or direct PD parameter. Therefore, it is under the necessity of doing make the relevant biomarkers which closed associate target drug. Since proteomic approaches can be used to identify drug-specific biomarker proteins, we also discuss how they may facilitate PK-PD modeling and simulation of other classes of drugs.

\section{Definition of PK-PD modeling and simulation}

The term PK-PD modeling and simulations refers to pharmacokinetic and pharmacodynamic data-driven exploratory analysis based on a mathematical/statistical model. PD response does not generally parallel plasma levels; therefore, models can help clinicians and researchers understand this relationship and how it changes as a function of administration and other variables. A model cannot be pre-specified fully before an experiment and must therefore be developed, or further refined, depending on experimental results. Modeling and simulation generally involves a combined analysis of data from several studies [9-11].

The discipline of modeling is data-driven, and it relies on multiple analyses of the same dataset in an iterative mode with successive and/or

*Corresponding author: Dr. Yoshikazu Matsuda, Clinical Pharmaceutics Educational Center, Nihon Pharmaceutical University, 10281 Komuro, Ina-machi, Saitama 362-0806, Japan, E-mail: yomatsuda@nichiyaku.ac.jp

Received August 12, 2011; Accepted September 17, 2011; Published September 19, 2011

Citation: Sako K, Haniu H, Hasegawa M, Doi H, Yano S, et al. (2011) The Application of Proteomics to PK-PD Modeling and Simulation. J Bioequiv Availab S2. doi:10.4172/jbb.S2-002

Copyright: (c) 2011 Sako K, et al. This is an open-access article distributed under the terms of the Creative Commons Attribution License, which permits unrestricted use, distribution, and reproduction in any medium, provided the original author and source are credited. 
competing models. It is possible to extrapolate beyond the boundaries of the design on which the models are defined. To accomplish this, models must express the explicit values of those boundaries and must incorporate current scientific understanding of the field in question in order to produce credible extrapolations [8,11]. A simulation model built using preclinical and clinical data should be considered a working model that will be refined as more data become available. Thus, PK-PD modeling and simulations are continuously updated throughout different stages of drug development and implementation to incorporate relevant new data. These updated modeling and simulations assist in formulating plans regarding how the next step in development should be performed. A full simulation model will typically consist of a number of submodels, which will include aspects such as dose (concentration) and inter- and intra-individual variability. Finally, well-defined modeling and simulations can be used to predict trial outcomes and curative effects through the use of information pertaining to hundreds of individual patients and from a number of different clinical situations and covariates [2]. An example of a PK analysis of a drug is shown in Figure 1.

\section{PK-PD modeling and simulation of antimicrobials in clinical settings}

PK aims to quantify the plasma level of an agent over time through the analysis of PK parameters. Alternatively, PD is used to quantify the activity of an antimicrobial agent by integrating its PK parameters with the MIC for a particular pathogen. From the PD point of view, antibiotics can be categorized based on their mode of bacterial killing and the presence or absence of a post-antibiotic effect (PAE), which is defined as the time required by the pathogen to resume normal growth following exposure to the agent $[12,13]$. Thus, the pattern of bacterial killing of an antibiotic can be either concentration-dependent if higher concentrations of the agent result in more extensive elimination of the pathogen, or time-dependent if the effectiveness of bacterial killing depends upon the duration of pathogen exposure to the agent. Based on these criteria, antimicrobial agents can be classified into two categories. The first category includes drugs that exhibit concentration-dependent killing in combination with a prolonged PAE (e.g., aminoglycosides, fluoroquinolones). The best predictors of efficacy for this class of agents are the peak concentration divided by the MIC $\left(\mathrm{C}_{\max } / \mathrm{MIC}\right.$, e.g., aminoglycosides) $[14,15]$ and/or the AUC for the first $24 \mathrm{~h}$ divided by

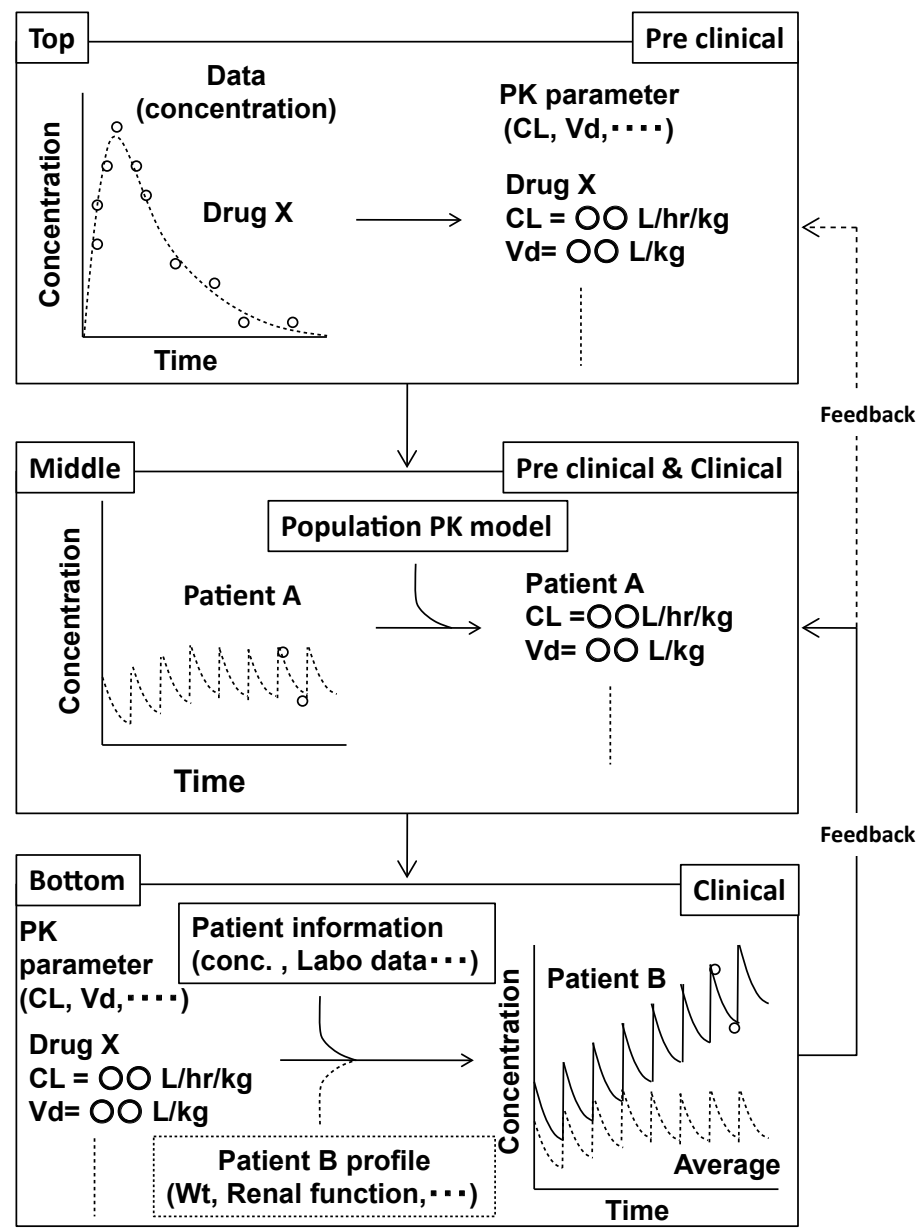

Figure 1: Concept of the feedback rope model of the relationship between preclinical data and clinical outcome. Top: Unique PK parameter of the objective drug is evaluated in preclinical situations. Middle: Individual patient PK parameter is evaluated in preclinical and clinical situations. Bottom: Decision regarding individual dosing regimen is made in clinical situation. ( $\mathrm{CL}=$ clearance; $\mathrm{Vd}=$ distribution volume; $\mathrm{Wt}=$ body weight $)$ 
the MIC ( $\mathrm{AUC}_{0-24} / \mathrm{MIC}$, e.g., fluoroquinolones) $[16,17]$. The second category of antimicrobial agents includes drugs that exhibit a timedependent pattern of killing and a minimal or moderate PAE (e.g., beta-lactams, macrolides, glycopeptides). For this category of agents, the percentage of a dosage interval in which the drug concentration exceeds the MIC (T > MIC, e.g., beta-lactams) [18] and/or the $\mathrm{AUC}_{0}$. ${ }_{24}$ /MIC (e.g., glycopeptides) $[19,20]$ are the parameters most strongly correlated with clinical efficacy (Figure 2).

In addition to the above classification, antibiotics can be classified as either hydrophilic or hydrophobic based on their ability to cross cellular membranes and by their resulting distribution volume [12,13]. The distribution volume of hydrophilic antibiotics is limited to the extracellular space and their plasma and interstitial concentrations may decrease due to fluid extravasations. These agents are inactive against intracellular bacteria, and are eliminated through the kidneys. Hydrophobic antibiotics have a large distribution volume, and dilution by interstitial fluid is less relevant with hydrophobic agents than with hydrophilic antibiotics. The hydrophobic antibiotics are active against intracellular bacteria and are primarily eliminated by the liver.

\section{Application of proteomics to PK-PD modeling and simulation}

Clinical PK-PD modeling and simulation of antimicrobial agents are possible because MIC values can be easily measured in the hospital laboratory. However, no such easily measured clinical markers are available for many other types of drugs. Although many researchers tried to find the PD parameter which associated non-antimicrobial agents (Table 1), these parameters were indirect parameters [21]. Therefore, it is necessary to find drug specific direct PD parameter.

During the last decade there has been growing interest in proteomics and system biology in general. A main focus has been exploring the technology to study complex multigenetic disease, to predict drug response, to individualize treatment and to discriminate between healthy and diseased individual [22-24]. We also had strongly interested in the proteomics approach for the toxicological- and pharmacological evaluation. Therefore, we thought that the proteomics approach may be available for find the direct PD parameter such as drug specific biomarker. In this section, we introduced our study results of carbon nanotubes (CNTs) which thought that the one of example in drug evaluation for bioresponse.

We previously evaluated the toxicological properties of carbon nanomaterials as part of a study aimed at enhancing their biocompatibility $[25,26]$. Using a proteomic approach, we found that carbon nanotubes (CNTs) induce minimal changes in cultured cells, and that they are not cytotoxic. Carbon black $(\mathrm{CB})$ is a type of carbon nanomaterial reported to induce an inflammatory response in vivo
Time-dependent antibiotics

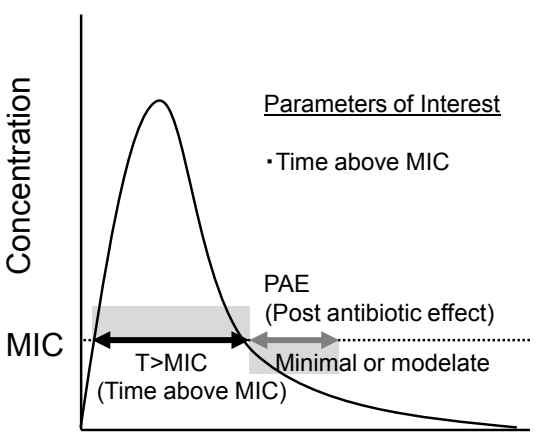

Time

\section{Concentration-dependent antibiotics}

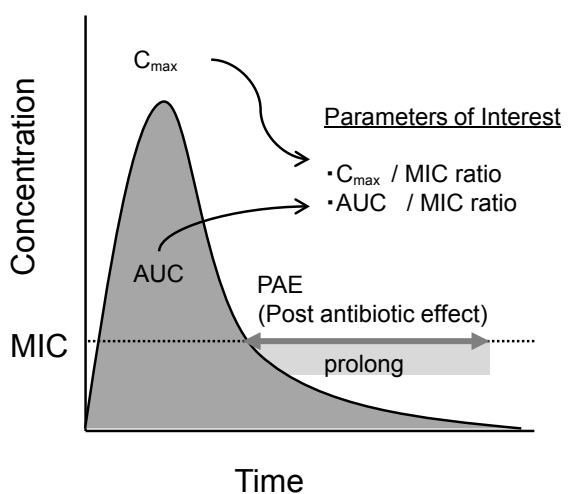

Figure 2: Principal PK-PD characteristics of antimicrobial drugs.

Left: Time-dependent antibiotics (beta-lactams, including penicillins and penems, glycopeptides, linezolid, macrolides, etc.). The time that the concentration of a drug remains above the MIC (T > MIC) is the PK-PD index correlating with efficacy. The post-antibiotic effect (PAE) can be absent (macrolides), minimal (betalactams, including penicillins and penems), or moderate (glycopeptides, linezolid).

Right: Concentration-dependent antibiotics (aminoglycosides, fluoroquinolones). The peak concentration/minimum inhibitory concentration ( $\left.\mathrm{C}_{\mathrm{max}} / \mathrm{MIC}\right)$ ratio and/or the area under the concentration-time curve at $24 \mathrm{~h} / \mathrm{MIC}\left(\mathrm{AUC}_{0-24} / \mathrm{MIC}\right)$ ratio are the best PK-PD modeling and simulation correlating with efficacy. Moreover, there is a prolonged PAE with the concentration-dependent antibiotics.

\begin{tabular}{|l|l|l|l|}
\hline \multicolumn{1}{|c|}{ Drug } & \multicolumn{1}{c|}{ Target Disease } & \multicolumn{1}{c|}{ PK } & survival time \\
\hline Docetaxel & non-small-cell lung cancer & cumulative AUC & BPRS \\
\hline Quetiapine & schizophrenia & plasma concentration & migraine improvement rate \\
\hline Naratriptan & migraine & effect compartment concentration & blood pressure, heart rate \\
\hline Ivabradine & angina pectoris & effect compartment concentration & pain score \\
\hline Pregabalin & neuropathic pain & $\begin{array}{l}\text { plasma concentration } \\
\text { (average) }\end{array}$ & uCTX \\
\hline Ibandronate & osteoporosis & DODR & fasting plasma glucose \\
\hline Gliclazide & diabetes & AUC & \\
\hline
\end{tabular}

BPRS : Brief Psychiatric Rating Scale Scores

DODR : Dose-Driving Rate

UCTX : urine and urinary excretion of the C-telopeptide of the a chain of type I collagen

Table 1: Typical examples include pharmacokinetic (PK) - pharmacodynamic (PD) modeling and simulation in non-antimicrobial agents [21] 
Citation: Sako K, Haniu H, Hasegawa M, Doi H, Yano S, et al. (2011) The Application of Proteomics to PK-PD Modeling and Simulation. J Bioequiv Availab S2. doi:10.4172/jbb.S2-002

Page 4 of 6

[27-29], and to influence cytokine and chemokine production and neurotransmission [30-32]. Cytotoxicity and changes in the expression of cytokines upon in vivo exposure to CB have been reported [29,33,34]. However, other reports indicate that $\mathrm{CB}$ exposure has no significant effect on cell proliferation, nor does it induce apoptosis or necrosis $[35,36]$. The current consensus is that CB may induce some biological responses, such as inflammation, but that there is little association between $\mathrm{CB}$ exposure and serious disease. In our experiments, $\mathrm{CB}$ had no effect on cell growth, and did not induce apoptosis or necrosis.

We used a proteomic approach to examine the effect of $\mathrm{CB}$ exposure on protein expression in cultured cells. Although CB exposure did not affect cell proliferation or induce cytotoxicity, it did lead to significant changes in the level of expression of 14 protein spots as determined using 2-dimensional gel electrophoresis. Using Matrix Assisted Laser Desorption/Ionization Time-of-Flight Mass Spectrometry (MALDITOF MS), a total of 10 proteins were identified within these 14 spots (Table 2). Although most of the identified proteins (7 out of 10) are associated with metabolism, proteins that are involved in development and cell death were also differentially expressed upon $\mathrm{CB}$ exposure (Table 3).

In another study, we found that although multi-wall carbon nanotubes (MWCNTs) do not inhibit cell proliferation or cause cytotoxicity in vitro, exposure to them does induce changes in the expression of some proteins involved in the stress response. Moreover, we found that expression of DJ-1, a biomarker of Parkinson's disease that has also been implicated in the development of cancer, changes after exposure to MWCNTs [25,37]. Our research suggests that proteomics is a promising means of identifying suitable biomarkers for use in PD modeling and simulations of these drugs.

On the other hands, there were in vitro and in vivo study of pharmacoproteomics in clinical setting carried out in recent years. Ghatpande et al. [38] reported in vitro protein profiling using twodimensional difference gel electrophoresis (2D-DIGE) on sickle red blood cell (RBC) membranes and identified a significant increase in predominantly anti-oxidant enzyme, protein repair and degradation components. The in vitro protein profiling system allowed to look at the same sickle RBC membrane from individual sickle cell anemia (SS) patients with and without drug of hydroxyurea (HU) exposure to identify dose dependent proteomic changes in vitro, which is difficult to achieve in an in vivo clinical setting. However, the in vitro system utilizes mature enucleated RBC that lack the capacity to synthesize new proteins and the in vitro proteomic changes identified mainly reflect post-translational modifications. Then, they had undertaken an in vivo proteomic analysis of sickle RBC membrane from of HU treated and untreated patients [39]. Since, in the in vivo system, the main target of $\mathrm{HU}$ is the bone marrow, protein changes at the level of protein expression as well as post-translational modification can be analyzed.

\begin{tabular}{|c|c|c|c|c|c|c|c|}
\hline spot No. & Protein Name & Theoretical MW & Theoretical pl & Score* & $\begin{array}{l}\text { Sequence } \\
\text { Coverage }\end{array}$ & Ratio & Accession No. \\
\hline 2 & transportin 1 & 103091 & 4.81 & 102 & $13 \%$ & 3.80 & Q92973 \\
\hline 5 & splicing factor $3 a$ subunit 3 & 59238 & 5.27 & 76 & $14 \%$ & 0.46 & Q12874 \\
\hline 6 & phosphogluconate dehydrogenase & 53745 & 6.80 & 71 & $12 \%$ & 0.47 & P52209 \\
\hline 8 & thioredoxin domain containing 5 & 44636 & 5.77 & 68 & $12 \%$ & 0.20 & Q8NBS9 \\
\hline 9 & spermine synthase & 24942 & 5.16 & 71 & $19 \%$ & 0.34 & P52788 \\
\hline 10 & annexin $\mathrm{A} 2$ & 38864 & 7.57 & 114 & $28 \%$ & 0.34 & P07355 \\
\hline 11 & otubain 1 & 31549 & 4.85 & 81 & $26 \%$ & 0.23 & Q96FW1 \\
\hline 12 & DnaJ homolog subfamily $\mathrm{C}$ member 8 & 29823 & 9.04 & 89 & $28 \%$ & 0.42 & O75937 \\
\hline 13 & actin related protein $2 / 3$ complex subunit 2 & 34454 & 6.84 & 71 & $20 \%$ & 0.49 & O15144 \\
\hline 14 & 14-3-3 protein $y$ & 28498 & 4.80 & 150 & $36 \%$ & 0.39 & P61981 \\
\hline
\end{tabular}

* Probability-based MOWSE score. Scores higher than 65 indicate the level of statistical significance at $p<0.05$.

Table 2: Proteins differentially expressed in cells exposed to carbon black. Proteins were identified using a proteomic approach involving 2-dimensional gel electrophoresis and MALDI-TOF MS. A total of 14 differentially expressed spots were detected, from which 10 proteins were identified. No proteins were identified from spots $1,3,4$, and 7 [25].

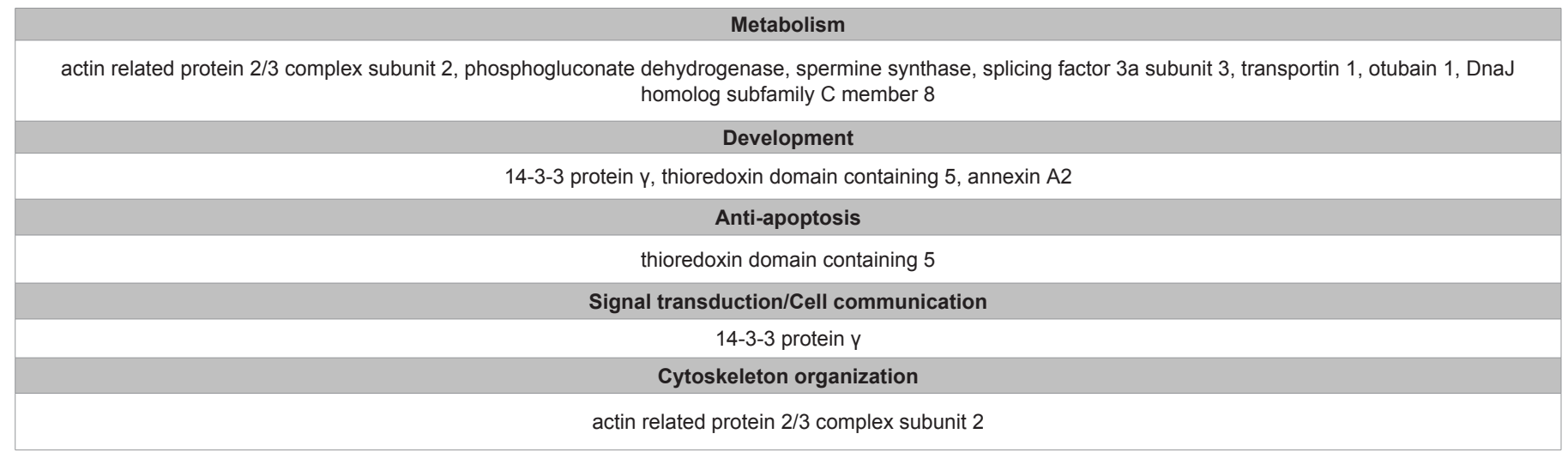

The protein functions were based on the Gene Ontology Annotation database.

Table 3: The primary functions of the proteins shown in Table 2 [25] 
However, these changes are a reflection of changes throughout erythropoiesis as well as after $\mathrm{RBC}$ maturation.

Andersson et al. [40] reported that the in vivo protein profile in short prepubertal children using a pharmaco-proteomic approach. Interestingly, their work was serum protein profiling using Surface-enhanced Laser Desorption/Ionization Time-of-Flight Mass Spectrometry (SELDI-TOF MS). Their used SELDI-TOF MS, a high-throughput technique which is suitable for analyzing large numbers of samples, in order to identify specific protein profile that are correlated with growth in response to treatment, and to get more insight into growth hormone dependent regulation of longitudinal growth. The challenge in proteomic analysis of serum is the broad range of expression levels between proteins with low and high abundance [41-43]. In order to partly overcome this problem, they used fractionated serum that was analyzed on different array surface in order to detect proteins in a larger area of the proteome. Moreover, Suehara et al. reported that the proteomic profile of synovial sarcoma using 2D-DIGE [44]. They identified 20 protein spots whose intensity were statistically different between a group of eight patients who were alive and continuously disease free for over five years and a group of five patients who died of the disease within two years post diagnosis.

These results suggest that proteomics is a promising tool in the search for drug-specific biomarker proteins that could be used in PK-PD modeling and simulation. Thus, by enabling examination of drug-induced changes in the expression of specific biomarker proteins, proteomic data could be used in PD analyses in much the same way that MICs are used in PD evaluations of antimicrobial drugs. We have therefore undertaken to identify drug-specific biomarker proteins using a proteomic approach and will attempting to clarify the relationship between identified biomarkers and target drugs.

Finally, there are problems when we think the proteomics application for PD parameter. First problem is how to detect PD parameter using proteomic approach. In clinical setting, there are many changes in patient such as drug effect, disease condition and compensatory change in patient body. Second problem is how to design the proteomics approach. Target sample of proteomics analysis may be varied by target drugs. For example, serum is a target sample for hormone drugs and tissue cells derived from patient is for anticancer drugs. Thus, we think that the further study will be needed for dissoluble above problems.

\section{Conclusion}

PK-PD modeling and simulation can be invaluable tools for use in the decision-making process during the crucial stages of drug development and in clinical settings. These may include decisions regarding compound selection, dose selection, study design, and patient population, all of which can impact the cost of drug development and treatment. Similarly, the optimization of therapeutic plans through the use of modeling and simulations leads to fewer instances of inappropriate application of pharmacotherapies and to improved treatment results.

The use of PK-PD modeling and simulation has already been successfully applied to the evaluation of antimicrobial agents because of the ease with which PD evaluations can be made. However, it is difficult to evaluate the PD of other classes of drugs using modeling and simulations because of the absence of suitable markers. Proteomics may be a means of overcoming this obstacle because it can be used to identify drug-specific biomarker proteins that can be applied to
PK-PD modeling and simulation. In a manner analogous to the way that simple PD parameter such as MICs are used in the PD analysis of antimicrobial agents, our research suggests that proteomic approaches could be used to evaluate changes in specific markers induced by other classes of drugs.

\section{Acknowledgements}

We thank Kizuna Hirahara and Midori Fujikawa at the Clinical Pharmaceutics Educational Center, Nihon Pharmaceutical University for their secretarial assistance. We also thank all pharmacists of Department of Pharmacy, Saitama Medical Center, Saitama Medical University, and Department of Pharmacy, Funabashi General Hospital for valuable discussion in clinical availability of PK-PD modeling and simulation.

\section{References}

1. Gobburru JV, Marroum PJ (2001) Utilization of pharmacokineticpharmacodynamic modeling and simulation in regulatory decision making. Clin Pharmacokinet 40: 863-892.

2. Burman CF, Hamren B, Olsson P (2005) Modeling and simulation to improve decision making in clinical development. Pharmaceut Statist 4: 47-58.

3. FDA (2004) Innovation and stagnation: challenge and opportunity for the critica path to new medicinal products.

4. Chang M, Chow SC, Pong A (2006) Adaptive design in clinical research issues, opportunities and recommendations. J Biopharm Stat 16: 299-309.

5. Biomarkers Definitions Working Group (2001) Biomarkers and surrogate endpoints in clinical trails: proposed definitions and conceptual framework. Clin Pharmacol Ther 69: 89-95.

6. Chaikin P, Rhodes GR, Bruno R, Rohatagi S, Natarajan C (2000) Pharmacokinetics/pharmacodynamics in drug development: an industrial perspective. J Clin Pharmacol 40: 1428-1438.

7. Gieschke R, Steimer JL (2000) Pharmacometrics: modelling and simulation tools to improve decision making in clinical drug development. Eur J Drug Metab Pharmacokinet 25: 49-58.

8. Colburn WA, Lee JW (2003) Biomarkers, validation and pharmacokineticpharmacodynamic modeling. Clin Pharmacokinet 42: 997-1022.

9. Aarons L, Karlsson MO, Mentré F, Rombout F, Steimer JL, et al. (2001) Role of modeling and simulation in Phase I drug development. Eur J Pharm Sci 13 115-122.

10. Parmigiani G (2002) Modelling in Medical DecisionMaking:A Bayesian Approach. John Wiley \& Sons, Ltd.

11. Csajka C, Verotta D (2006) Pharmacokinetic-pharmacodynamic modeling: history perspectives. J Pharmacokin Pharmacodyn 33: 227-279.

12. Frimodt-Moller $\mathrm{N}$ (2002) How predictive is PK/PD for antibacterial agents? Int Antimicrob Agents 19: 333-339.

13. Scaglione F, Paraboni L (2008) Pharmacokinetics/pharmacodynamics of antibacterials in the Intensive Care Unit: setting appropriate dosing regimens. Int J Antimicrob Agents 32: 294-301.

14. Moore RD, Lietinan PS, Smith CR (1987) Clinical response to aminoglycoside therapy : importance of the ratio of peak concentration minimal inhibitory concentration. J infect Dis 155: 93-99.

15. Moore RD, Smith CR, Liotman PS (1984) Association of aminoglycoside plasma levels with therapeutic outcome in gram-negative pneumonia. Am J Med 77: 657-662.

16. Drusano GL, Johnson DE, Rosen M, Standiford HC (1993) Pharmacodynamics of a fluoroquinolone antimicrobial agent in a neutropenic rat model of Pseudomonas sepsis. Antimicrob Agents Chemother 37: 483-490.

17. Preston SL, Drusano GL, Berinan AL, Fowler CL, Chow AT, et al. (1998) Pharmacodynamics of fuvofloxacin:a now paradigm for early clinical trials. JAMA 279: 125-129.

18. Vogehnan B, Gudmundsson S, Leggett J, Turnidge J, Ebert S, et al. (1988) Correlation of antimicrobial pharmacokinetic parameters with therapeutic efficacy in an animal model. J Infect Dis 158: 831-847.

19. Knudsen JD, Fuursted K, Espersen F, Frimodt-Møller N (1997) Activities of vancomycin and teicoplanin against penicillin-resistant pneumococci in vitro 
Citation: Sako K, Haniu H, Hasegawa M, Doi H, Yano S, et al. (2011) The Application of Proteomics to PK-PD Modeling and Simulation. J Bioequiv Availab S2. doi:10.4172/jbb.S2-002

and in vivo and correlation to pharmacokinetic parameters in the mouse peritonitis model. Antimicrob Agents Chemother 41: 1910-1915

20. Moise-Broder PA, Forrest A, Birmingham MC, Schentag JJ (2004) Pharmacodynamics of vancomycin and other antimicrobials in patients with Staphylococcus aureus lower respiratory tract infections. Clin Pharmacokine 43: $925-942$

21. Nakade S (2005) Lecture note : paradigm shift in drug developments usefulness of PK/PD-(2) Modeling and simulation in clinical trial. Newsletter 20: 26-31.

22. Daly AK (2007) Individualized drug therapy. Curr Opin Drug Discov Devel 10 29-36

23. Rosenfeld RG (2007) Pharmacogenomics and pharmacoproteomics in the evaluation and management of short stature. Eur J Endocrinol 157: S27-31.

24. Weber WW (2001) Pharmacogenetic tactics and strategies: implications for paediatrics. Paediatr Drugs 3: 863-881.

25. Haniu H, Matsuda Y, Takeuchi K (2009) Potential of a novel safety evaluation of nanomaterials using a protemic approach. J Health Sci 55: 428-434.

26. Haniu H, Matsuda Y, Takeuchi K, Kim YA, Hayashi T, et al. (2010) Proteomicsbased safety evaluation of multi-walled carbon nanotubes. Toxicol Appl Pharmacol 242: 256-262.

27. Brown DM, Stone V, Findlay P, MacNee W, Donaldson K (2000) Increased inflammation and intracellular calcium caused by ultrafine carbon black is independent of transition metals or other soluble components. Occup Environ Med 57: 685-691.

28. Li XY, Brown D, Smith S, MacNee W, Donaldson K (1999) Short-term inflammatory responses following intratracheal instillation of fine and ultrafine carbon black in rats. Inhal Toxicol 11: 709-731.

29. Wilson MR, Lightbody JH, Donaldson K, Sales J, Stone V (2002) Interactions between ultrafine particles and transition metals in vivo and in vitro. Toxicol Appl Pharmacol 184: 172-179.

30. Tin-Tin-Win-Shwe, Yamamoto S, Kakeyama M, Kobayashi T, Fujimaki H (2005) Effect of intratracheal instillation of ultrafine carbon black on proinflammatory cytokine and chemokine release and mRNA expression in lung and lymph nodes of mice. Toxicol Appl Pharmacol 209: 51-61.

31. Tin-Tin-Win-Shwe, Mitsushima D, Yamamoto S, Fukushima A, Funabashi T, et al. (2008) Changes in neurotransmitter levels and proinflammatory cytokine mRNA expressions in the mice olfactory bulb following nanoparticle exposure. Toxicol Appl Pharmacol 226: 192-198.
32. Tin-Tin-Win-Shwe, Yamamoto $S$, Ahmed S, Kakeyama M, Kobayashi T, et al. (2006) Brain cytokine and chemokine mRNA expression in mice induced by intranasal instillation with ultrafine carbon black. Toxicol Lett 163: 153-160.

33. Herzog E, Casey A, Lyng FM, Chambers G, Byrne HJ, et al. (2007) A new approach to the toxicity testing of carbon-based nanomaterials-the clonogenic assay. Toxicol Lett 174: 49-60.

34. Soto K, Garza KM, Murr LE (2007) Cytotoxic effects of aggregated nanomaterials. Acta Biomater 3: 351-358.

35. Bottini M, Bruckner S, Nika K, Bottini N, Bellucci S, et al. (2006) Multi-walled carbon nanotubes induce T lymphocyte apoptosis. Toxicol Lett 60: 121-126.

36. Lam CW, James JT, McCluskey R, Hunter RL (2004) Pulmonary toxicity of single-wall carbon nanotubes in mice 7 and 90 days after intratracheal instillation. Toxicol Sci 77: 126-134.

37. Haniu H, Matsuda Y, Usui Y, Aoki K, Shimizu M, et al. (2011) Toxicoproteomic evaluation of carbon nanomaterials in vitro. J Proteomics [Epub ahead of print]

38. Ghatpande SS, Choudhary PK, Quinn CT, Goodman SR (2008) Pharmacoproteomic study of hydroxyurea-induced modifications in the sickle red blood cell membrane proteome. Exp Biol Med 233: 1510-1517.

39. Ghatpande SS, Choudhary PK, Quinn CT, Goodman SR (2010) In vivo pharmaco-proteomic analysis of hydroxyurea induced changes in the sickle red blood cell membrane proteome. J Proteomics 73: 619-626.

40. Andersson B, Hellgren G, Nierop AF, Hochberg Z, Albertsson-Wikland K (2009) Proteins related to lipoprotein profile were identified using a pharmacoproteomic approach as markers for growth response to growth hormone $(\mathrm{GH})$ treatment in short prepubertal children. Proteome Sci 7: 40.

41. Anderson NL, Anderson NG (2002) The human plasma proteome: history, character, and diagnostic prospects. Mol Cell Proteomics 11: 845-867.

42. Corthals GL, Wasinger VC, Hochstrasser DF, Sanchez JC (2000) The dynamic range of protein expression: a challenge for proteomic research. Electrophoresis 21: 1104-1115.

43. Thadikkaran L, Siegenthaler MA, Crettaz D, Queloz PA, Schneider P, et al (2005) Recent advances in blood-related proteomics. Proteomics 5: 3019 3034

44. Suehara Y, Tochigi N, Kubota D, Kikuta K, Nakayama R, et al. (2005) Secernin-1 as a novel prognostic biomarker candidate of synovial sarcoma revealed by proteomics. J Proteomics 74: $829-842$.

This article was originally published in a special issue, PK/PD: Drug

Discovery and Development handled by Editor(s). Dr. Bolin Geng, AstraZeneca R\&D Boston, USA; Dr. Jian Li, Monash University, Australia 